\section{Chronic diseases in Nyeri, Kenya: a study of knowledge and perceptions}

\author{
Michael A.T. Freiberg,' \\ Nelson O. Onyango, ${ }^{1}$ \\ Stephanie J. Ashbaugh, ${ }^{1}$ Khanjan Mehta ${ }^{2}$ \\ 'Department of Family and Community \\ Medicine, Pennsylvania State University \\ College of Medicine, Hershey, PA; \\ ${ }^{2}$ Humanitarian Engineering and Social \\ Entrepreneurship (HESE) Program, \\ Pennsylvania State University College of \\ Engineering, University Park, PA, USA
}

\section{Abstract}

The burden of chronic, non-communicable disease such as diabetes, cardiovascular disease, and cancer is growing in many developing countries including Kenya. The use of community health workers is an important tool to improve the access to care and education in rural areas. This study aims to understand the knowledge and perceptions among the general population regarding three chronic diseases diabetes, hypertension, and cancer - in Nyeri, Kenya. Standardized, open-ended interviews were conducted with 200 participants. This study shows that most individuals interviewed are familiar with these three diseases; however, knowledge varied among individuals with many having significant gaps in knowledge. These results are consistent with previous studies from this region and will inform future education directed at community health workers and the general population.

\section{Introduction}

The burden of chronic, non-communicable disease continues to grow throughout Africa and across the developing world. Although the most recent data from the World Health Organization show that the majority of deaths in Africa between 2000-2012 were from communicable disease at $61.7 \%$ compared to $28.6 \%$ for non-communicable disease and 9.8\% from injury, this distribution is quickly changing. ${ }^{1}$ By $2020,73 \%$ of deaths worldwide are expected to be due to non-communicable disease with $79 \%$ of these deaths occurring in developing countries. $^{2-4}$ Many factors have been attributed to this rise including increased lifespan, modernization, globalization, poverty, and changing lifestyle. ${ }^{4,5}$ This problem is compounded by the fact that many health care systems are underfunded and ill-prepared to handle the current state of non-communicable disease and the inevitable rise over the coming years will put additional burden on these systems. Of the money that is spent on healthcare in Africa, most of which is from foreign sources, it has been estimated that $80 \%$ is spent on communicable diseases including HIV, malaria, and tuberculosis. ${ }^{67}$ Many health ministries acknowledge this disparity; however, little has been done to change this imbalance. This imbalance results in healthcare systems that are poorly prepared to handle non-communicable disease and a population that is uninformed of the risks, treatment, and prevention. ${ }^{8,9}$

A multifactorial approach will be necessary to improve the outlook of non-communicable disease in the developing world and important components will include improving access to health care services and improving education. One common approach to accomplish both of these goals in many countries in Africa and across the developing world is the community health worker (CHW) model. Under this model, community members are selected to serve as the first point of contact for health care and education. CHWs are members of the communities that they serve; they know the local language, culture, and people well and are thus in a good position to enact change.

The ability of CHWs to enact change and improve health care for medically underserved patients in both high and low income countries has been well documented. ${ }^{10-14}$ Their impact, however, is somewhat limited by their lack of medical knowledge and experience. ${ }^{15}$ Most have no formal medical education are often chosen because of their position in the community rather than the amount of medical knowledge they have.

In order to improve the positive impact of CHWs, an effective education program is necessary and should focus on the knowledge gaps and cultural attitudes of the community members in each region. ${ }^{10}$ Much work has already been done to understand these knowledge gaps in Kenya, Africa and the developing world and most studies demonstrate that knowledge is lacking. ${ }^{16-19}$ The focus for this study is on three non-communicable diseases - hypertension, diabetes, and cancer.

Hypertension and diabetes, both significant risk factors for cardiovascular disease, are common in Kenya. ${ }^{16-18}$ As such, more effective prevention and treatment of these conditions represents an excellent opportunity to reduce the overall burden of chronic disease. One study, which estimated the prevalence of hypertension to be $21 \%$ in rural Kenya found that $83 \%$ of participants with hypertension were not aware of their condition and only $3 \%$ were being successfully treated. ${ }^{17}$ Another study in Mombasa, the second largest city in Kenya, found the prevalence of hypertension to
Correspondence: Michael A.T. Freiberg, Department of Family and Community Medicine, Pennsylvania State University College of Medicine, Hershey, PA, USA.

Tel: +1.717.4334804.

E-mail: michael.a.t.freiberg@gmail.com

Key words: Kenya; Diabetes; Hypertension; Cancer; Community health worker.

Acknowledgments: the authors would like to acknowledge Dr. Timothy Irwin for assistance with interviews conducted in this study. We would also like to acknowledge Mr. David Ndirangu Kihara who served as our interpreter in Kenya.

Contributions: the study was designed by MF and NO with the advice and guidance of SA and KM The surveys were conducted by MF, NO, and SA. The data analysis was done by MF and NO. The paper was written by MF and NO and critically analyzed by SA and KM.

Conflict of interest: the authors declare no potential conflict of interest.

Note: this research was approved by the institutional review board of the Penn State University and informed consent was obtained verbally from each subject.

Received for publication: 6 December 2016. Revision received: 30 October 2016. Accepted for publication: 11 November 2016.

This work is licensed under a Creative Commons Attribution 4.0 License (by-nc 4.0).

(C) Copyright M.A.T. Freiberg et al., 2016

Licensee PAGEPress, Italy

Healthcare in Low-resource Settings 2016; $4: 5669$ doi:10.4081/hls.2016.5669

be even higher at $32 \%$ with only $53 \%$ of these individuals being aware of their condition and only $23 \%$ familiar with the causes and treatments. ${ }^{16}$ Knowledge of diabetes is also lacking. At an estimated prevalence of $3.58 \%$ and as high as $12 \%$ in some urban populations, diabetes is common in Kenya. ${ }^{18}$ One study found that $73 \%$ of people interviewed across four regions of Kenya had poor knowledge of diabetes and not surprisingly, education was correlated with a higher knowledge of diabetes. ${ }^{19}$

Cancer, which is more complicated to treat than other chronic diseases, is a target for primary prevention. Cervical cancer, the most common cancer for women in Kenya, has been the most studied since it is largely preventable with screening, vaccination, and safe sexual practices. One study in rural Kenya showed that $35 \%$ of respondents had never even heard of cervical cancer and only slightly more than half had ever heard of a Pap smear. ${ }^{20}$ Another study among patients in a government hospital 
in Nairobi found similar results with only half of patients knowing about cervical cancer and only $32 \%$ knowing about the Pap smear. ${ }^{21}$

The present study aims to understand the current state of knowledge and perceptions of these three chronic diseases - hypertension, diabetes, and cancer - in Nyeri, Kenya. A deeper understanding of the knowledge and perceptions of diabetes, hypertension, and cancer in this region, will guide and focus future education of both CHWs and community members on non-communicable disease.

\section{Materials and Methods}

\section{Site selection}

Nyeri is a city located in the central highland region of Kenya, approximately $100 \mathrm{~km}$ north of country's capital, Nairobi. The city and surrounding villages has a population of approximately 125,000 people. ${ }^{22}$ The local language in Nyeri is Kikuyu; however, both English and Swahili are widely spoken. This site was chosen because there is convenient access to both rural and urban populations.

\section{Study design}

Standardized, open-ended interviews were conducted with one interviewer asking questions to one subject. Interviews were conducted either completely in English, in English with the assistance of a Kikuyu-speaking interpreter, or in Swahili depending on the preference of the participant. After recording the age and gender of the participant, the participant was asked four standardized, openended questions about each of the three diseases being studied - diabetes, high blood pressure, and cancer: i) What is (disease) and what are some signs and symptoms? ii) How does someone get (disease)? iii) How is (disease) treated? iv) How can (disease) be prevented?

Finally, each participant was asked an additional question Do you have anything you would like to share or any stories about how these diseases have affected yourself, your friends, your family, or someone in your community?

Each participant was allowed to answer each question as thoroughly as he wished and the next question was asked only when the subject indicated he was finished with that question or began giving repetitive information. During the interview, thorough handwritten notes were taken of each participant's response and the notes were later transcribed electronically.

\section{Participant selection and sample size}

Interviews were conducted during daylight hours between 0900 to 1500 at multiple loca- tions in both the urban center of Nyeri and in surrounding rural areas in order to get a wide range of participants. To identify participants, the interviewers walked through the community and asked people if they would be willing participate. Only individuals 18 years of age or older were allowed to participate and informed consent was obtained verbally prior to each interview. Individuals under 18 or who did not agree to participate in the survey were not included in the analysis. A total of two-hundred interviews was conducted and the sample size was determined based on a saturation of responses.

\section{Data analysis}

After reviewing the transcripts of all responses, a list of reported response features relevant to each disease was developed. These response features included elements that suggest an understanding of the disease etiology or pathophysiology (i.e. participant knows that diabetic patients have blood sugar that is too high or too low), signs and symptoms of the disease (i.e. hypertension causes headaches), causes of the disease (i.e. cancer is caused by tobacco use), and treatment of the disease (i.e. diabetes can be treated with insulin). Each subject's disease-specific response was then analyzed for the presence or absence of each specific response feature. Response features with the same meaning, despite exact wording, were considered equivalent (i.e. a participant who said that diabetes causes polyuria was given credit for the increased urination response feature). Response features that could be considered both a cause and treatment (i.e. lack of exercise causes hypertension or exercise is a treatment for hypertension) were considered equivalent responses.

\section{Results}

\section{Subject population}

Not everyone who was invited to participate in an interview agreed to participate and only data from individuals who completed interviews is included in the analysis. A total of 200 individuals completed interviews including 99 females and 101 males. Subjects had a mean age of 37.1 years old with a standard deviation of 14.4 years.

Interviews were conducted in nine different localities across Nyeri County that included both rural and urban settings. Most interviews lasted between five and fifteen minutes depending on the length of answers given by the subject.

Of the participants interviewed, 8 people (4\%) reported having no formal education, 166 people $(83 \%)$ reported having completed at least Class 8 (equivalent to $8^{\text {th }}$ grade in the
United States), 104 people (52\%) reported finishing at least From 4 (equivalent to $12^{\text {th }}$ grade in the United States), and 22 people (11\%) reported having completed at least some postsecondary education (Table 1).

\section{Disease-specific criteria}

A summary of participant's responses is presented for diabetes (Table 2), hypertension (Table 3), and cancer (Table 4).

\section{Discussion}

Non-communicable diseases such as diabetes, hypertension and cancer represent a significant and growing burden to the health of people in the developing world. ${ }^{1-4}$ While the barriers to decreasing the burden of chronic disease are multifactorial, education of both community members and community health workers will play an important role. This study identified significant knowledge gaps in this region, consistent with prior studies. . $6,17,19-21,23$

While overall knowledge of diabetes was lacking in this study population, the most notable knowledge gaps identified related to lifestyle risk factors and signs and symptoms. While most of the research on diabetes knowledge has focused on people with the disease, our findings are consistent with previous studies sub-Saharan Africa. ${ }^{19,23}$ Since symptom identification can be a motivation for people to seek care, education should focus on recognizing signs and symptoms of diabetes, especially among CHWs who are well situated to identify those at risk.

Table 1. The highest level of education that the two-hundred surveyed completed.

\begin{tabular}{lcc} 
Highest education & n & $\%$ \\
level completed & & \\
No formal education & 8 & 4 \\
Class 1 & 0 & 0 \\
\hline Class 2 & 1 & 1 \\
Class 3 & 0 & 0 \\
\hline Class 4 & 2 & 1 \\
Class 5 & 4 & 2 \\
\hline Class 6 & 2 & 1 \\
Class 7 & 17 & 9 \\
\hline Class 8 & 43 & 22 \\
Form 1 & 1 & 1 \\
\hline Form 2 & 12 & 6 \\
Form 3 & 6 & 3 \\
\hline Form 4 & 82 & 41 \\
Post-secondary education & 22 & 11 \\
\hline N=200, percentages were calculated using a denominator of 200.
\end{tabular}


This study also identified a poor understanding of hypertension among participants. Most notably, many of the signs and symptoms named were overemphasized. A variety of responses were given including headache, dizziness and fainting, fatigue, difficulty breathing, swelling, anger, and vision changes. While these are all potential signs and symptoms of hypertension, no participant said that hypertension can be asymptomatic. This represents a significant disconnect since hypertension commonly presents asymptomatically and other studies from Kenya suggest that the majority of people with hypertension are unaware that they have the condition. ${ }^{16,17}$ Education on the importance of regular screening should be emphasized along with efforts to improve screening of hypertension through home and community based programs is already underway. ${ }^{24,25}$ Additionally, respondents seemed to overemphasize stress as a cause hypertension when other factor such as diet, lack of exercise, and obesity are more significant contributors. Education to modify diet and increase exercise in a culturally relevant way represents an additional opportunity to prevent and treat hypertension.

Compared to diabetes and hypertension, cancer is more complicated and expensive to treat. The most cost-effective interventions to reduce the burden of cancer will focus on primary prevention rather than treatment. ${ }^{26}$ While slightly over half of respondents were able to name at least one type of cancer, knowledge of risk factors was lacking. For example, only six people (3\%) surveyed mentioned that cancer can be caused by sexual activity, or by a sexually transmitted infection, even though cervical cancer is the most common cancer among women in Kenya and is almost always caused by an infection with the sexually transmitted human papilloma virus (HPV)..$^{27,28}$ In addition, no one mentioned Pap smears specifically as a way to prevent or screen for cervical cancer. This finding is consistent with the results from other studies in Kenya which demonstrated that many women surveyed in both rural and urban hospitals had not even heard about cervical cancer or were not able to describe anything about the disease including prevention or treatment. ${ }^{20,21}$ While this may have been due to the cultural taboo of discussing sex, it is more likely due to the fact that widespread Pap smear programs or HPV vaccination programs have not yet begun in Kenya. Additionally, only $20 \%$ of people identified smoking or tobacco use as a cause of cancer. While tobacco use is only attributed to $6 \%$ of cancer deaths in Africa compared to about $20 \%$ of cancer deaths worldwide, most likely because of the relatively low life expectancy and low smoking prevalence, it is increasing in some parts of Africa. ${ }^{26}$ Education on the risks of tobacco represents an additional opportuni- ty for cancer prevention.

A cultural belief about cancer that several participants (4\%) mentioned was that cancer is a curse. Specifically, one respondent explained that going against your parent's requests after they had died, for example: selling their land against their wishes could give you a curse that causes cancer. Some participants also held the cultural belief that cancer is brought about by mimicking values and practices of the Western world such as the Western diet and vaccinations. Although relatively uncommon among our participants, these beliefs are an additional barrier to cancer prevention and treatment.

Our data does have some limitations and potential biases. Due to logistical limitations, random sampling was not feasible in this study

Table 2. Total number of individuals providing each response (n) when asked four questions related to diabetes: What is diabetes and what are some signs and symptoms? How does someone get diabetes? How is diabetes treated? How can diabetes be prevented?

\begin{tabular}{lcc} 
Response & n & $\%$ \\
Blood sugar is too high or too low & 58 & 29 \\
Signs and symptoms & & 18 \\
$\quad$ Fatigue & 35 & 15 \\
Increased urination & 29 & 14 \\
Dizziness or fainting & 28 & 11 \\
Increased thirst & 22 & 8 \\
Weight loss & 16 & 6 \\
Increased appetite & 11 & 6 \\
Vision changes & 11 & 6 \\
Poor wound healing & 12 & \\
Causes & & 68 \\
Poor diet & 135 & 15 \\
Inherited from family & 30 & 12 \\
Lack of exercise & 24 & 4 \\
Obesity & 7 & 4 \\
Alcohol Use & 7 & 3 \\
Tobacco use & 5 & \\
Treatment & & 44 \\
Any medication (pills and/or insulin) & 87 & 38 \\
Pills & 75 & 18 \\
Insulin & 35 & \\
\hline
\end{tabular}

$\mathrm{N}=200$, percentages were calculated using a denominator of 200 .

Table 3. Total number of individuals providing each response (n) when asked four questions related to hypertension: What is high blood pressure and what are some signs and symptoms? How does someone get high blood pressure? How is high blood pressure treated? How can high blood pressure be prevented?

\begin{tabular}{lcc} 
Response & n & \% \\
A disease of the heart of blood vessels & 75 & 38 \\
Signs and symptoms & & 24 \\
Headache & 48 & 15 \\
Dizziness or fainting & 30 & 12 \\
Fatigue & 23 & 8 \\
Difficulty breathing & 15 & 8 \\
Swelling in hands or feet & 15 & 6 \\
Anger or irritability & 12 & 4 \\
$\quad$ Vision changes & 8 & \\
Causes & & 67 \\
$\quad$ Stress & 134 & 33 \\
Poor diet & 65 & 12 \\
Lack of exercise & 24 & 9 \\
Depression & 17 & 6 \\
Obesity & 11 & 8 \\
Inherited from family & 16 & 3 \\
Too much dietary salt & 5 & 50 \\
Treatment & & \\
Medications & 99 & \\
\hline
\end{tabular}


Table 4. Total number of individuals providing each response (n) when asked four questions related to cancer: What is cancer and what are some signs and symptoms? How does someone get cancer? How is cancer treated? How can cancer be prevented?

\begin{tabular}{lcc} 
Response & n & $\%$ \\
Named at least one type of cancer & 102 & 51 \\
Causes & & \\
$\quad$ Tobacco use & 40 & 20 \\
Inherited from family & 37 & 19 \\
Diet & 35 & 10 \\
Pesticides and other chemicals in food & 19 & 7 \\
Alcohol & 14 & 7 \\
Infectious & 13 & 5 \\
Unpreventable & 10 & 5 \\
Stress & 9 & 4 \\
Curse & 8 & 3 \\
Unsafe sex or HPV & 6 & 3 \\
Dirty living conditions or lack of hygiene & 5 & 2 \\
$\quad$ Solar radiation or x-rays & 3 & \\
Treatment & & 37 \\
Chemotherapy & 73 & 36 \\
Early detection & 72 & 29 \\
Surgery & 58 & 11 \\
Radiation therapy & 21 & 9 \\
Untreatable & 17 & \\
\hline
\end{tabular}

HPV, human papilloma virus. $\mathrm{N}=200$, percentages were calculated using a denominator of 200 .

and a relatively low sample population was used. Interviews were conducted in the community during daytime hours from 0900 to 1500 and this likely biased the sample toward certain demographics who were available to participate during this time. In addition, many people who were approached to participate in this survey declined to participate. Still, there is a wide range of ages and education levels in the people who did participate so this survey likely still provides a representative sample. One additional limitation was the manner that questions were asked. Since questions were open-ended, direct recollection was required by the subjects being interviewed.

\section{Conclusions}

There are many gaps and misconceptions about non-communicable disease in this region. Based on the knowledge deficiencies identified in this study, future public health and CHW educational programs should have an emphasis on preventative measures and lifestyle factors that contribute to each disease. Specifically, for diabetes the importance of diet and exercise should be targeted. Community health workers should be educated on appropriate dietary factors that contribute to the disease, with an additional emphasis on the signs and symptoms of diabetes. For hypertension, it will be important to emphasize that it is often an asymptomatic disease so screening is necessary despite the absence of symptoms. For cancer, smoking cessation, and moderate alcohol use are important concepts to emphasize. Safe sex practices are also important to prevent cervical cancer as well as other sexually transmitted disease. While it is also necessary to educate women on the importance of screening and vaccination to prevent cervical cancer this will likely have little effect until such services are widely available.

\section{References}

1. World Health Organization. Global causes of death. Geneva: World Health Organizaion; 2014.

2. Kearney PM, Whelton M, Reynolds K, et al. Global burden of hypertension: analysis of worldwide data. Lancet 2005;365:217-23.

3. World Health Organizaion. The world health report 2002. Geneva: World Health Organizaion; 2002.

4. World Health Organizaion. Preventing chronic disease: a vital investment. Geneva: World Health Organizaion; 2005.

5. McCormack VA, Schüz J. Africa's growing cancer burden: environmental and occupational contributions. Cancer Epidemiol 2012;36:1-7.

6. Ejughemre U. Donor support and the impacts on health system strengthening in sub-Saharan Africa: assessing the evidence through a review of the literature. Am J Public Heal Res 2013;1:146-51.

7. World Health Organizaion. The work of WHO in the African Region. Geneva: World Health Organizaion; 2007.

8. de-Graft Aikins A, Unwin N, Agyemang C, et al. Tackling Africa's chronic disease bur- den: from the local to the global. Global Health 2010;6:5.

9. Mensah GA. Epidemiology of stroke and high blood pressure in Africa. Heart 2008;94:697-705.

10. Mwai G, Mburu G, Torpey K, et al. Role and outcomes of community health workers in HIV care in sub-Saharan Africa: a systematic review. J Int AIDS Soc 2013;16:1-14.

11. Haines A, Sanders D, Lehmann U, et al. Acheiving child survival goals: potential contribution of community health workers. Lancet 2007;369:2121-31.

12. Gilmore B, McAuliffe E. Effectiveness of community health workers delivering preventive interventions for maternal and child health in low- and middle-income countries: a systematic review. BMC Public Health 2013;13:847.

13. Lewin S, Munabi-Babigumira S, Glenton $\mathrm{C}$, et al. Lay health workers in primary and community health care for maternal and child health and the management of infectious diseases. Cochrane database Syst Rev 2010;CD004015.

14. Swider SM. Outcome effectiveness of community health workers: an integrative literature review. Public Health Nurs 2002;19:11-20.

15. Kane S, Kok M, Ormel H, et al. Limits and opportunities to community health worker empowerment: a multi-country comparative study. Soc Sci Med 2016;164:27-34.

16. Jenson A, Omar AL, Omar MA, et al. Assessment of hypertension control in a district of Mombasa, Kenya. Glob Public Health 2011;6:293-306.

17. Hendriks ME, Wit FWNM, Roos MTL, et al. Hypertension in sub-Saharan Africa: cross-sectional surveys in four rural and urban communities. PLoS One 2012;7:e32638.

18. International Diabetes Federation. IDF Atlas, Sixth Edition. Brussels: International Diabetes Federation; 2013.

19. Maina WK, Ndegwa ZM, Njeng EW, Muchemi EW. Knowledge, attitude , and practices related to diabetes among community members in four provinces in Kenya : a cross-sectional study. African J Diabetes Med 2011;19:15-8.

20. Gatune JW, Nyamongo IK. An ethnographic study of cervical cancer among women in rural Kenya: is there a folk causal model? Int J Gynecol Cancer 2005;15:104959.

21. Gichangi P, Estambale B, Bwayo J, et al. Knowledge and practice about cervical cancer and Pap smear testing among patients at Kenyatta National Hospital, Nairobi, Kenya. Int $\mathbf{J}$ Gynecol Cancer 2003;13:827-33.

22. Open data portal. 2009 Census Vol 1 Table 3 Rurual and urban population. Open data 
portal. Open Kenya. Available from: https://www.opendata.go.ke/Population/20 09-Census-Vol-1-Table-3-Rural-andUrban-Populati/e7c7-w67t

23. Kiawi E, Edwards R, Shu J, et al. Knowledge, attitudes, and behavior relating to diabetes and its main risk factors amoung urban residnets in Cameroon: a qualitative survey. Ethn Dis 2006;16:503-9.

24. Pastakia SD, Ali SM, Kamano JH, et al.
Screening for diabetes and hypertension in a rural low income setting in western Kenya utilizing home-based and community-based strategies. Global Health 2013;9:21.

25. Lackey JD, Suffian S, Dzombak R, Mehta K. Demonstrating demand for preventive health services in rural Kenya. J Humanit Eng 2015;3:8-16.

26. Jemal A, Bray F, Forman D, et al. Cancer burden in Africa and opportunities for prevention. Cancer 2012;118:4372-84.

27. Walboomers J, Jacobs M, Manos MM, et al. Human papillomavirus is a necessary cause of invasive cervical cancer worldwide. J Pathol 1999;19:12-9.

28. World Health Organization. The global burden of disease. 2004 Update. Geneva: World Health Organizaion; 2008. Available from: http: 